\title{
Extracellular Synthesis of Copper Nanoparticles using Culture Supernatants of Salmonella typhimurium
}

\section{HAMID REZA GHORBANI*, FERDOS PARSA MEHR and AZIN KHANIYANI POOR}

Department of Chemical Engineering, Qaemshahr Branch, Islamic Azad University, Qaemshahr, Iran.

${ }^{*}$ Corresponding author E-mail: hamidghorbani6@ gmail.com

http://dx.doi.org/10.13005/ojc/310165

(Received: November 30, 2014; Accepted: January 10, 2015)

\begin{abstract}
In the recent years, the synthesis of metal nanoparticles has received much attention due to its wide range of applications in various fields. In this study copper nanoparticles were synthesized using salmonella typhimurium. Biosynthesis of copper nanoparticles was achieved by addition of culture supernatant with aqueous copper nitrate solution $(1 \mathrm{mM})$. Copper nanoparticles having average diameter $49 \mathrm{~nm}$ have been obtained. The reaction mixture exhibit a peak at $565 \mathrm{~nm}$ corresponding to the Plasmon absorbance of copper nanoparticles by UV-Vis Spectroscopy. The nanoparticles were characterized by dynamic light scattering (DLS), and Scanning Electron Microscopy (SEM). The process of reduction is extracellular, which makes it an easier method for the synthesis of copper nanoparticles.
\end{abstract}

Key words: Salmonella typhimurium, Biosynthesis, Copper nanoparticles, culture supernatants.

\section{INTRODUCTION}

In the recent decades, metallic nanoparticles have been widely used in different industries due to their wide range of applications'. Metal nanoparticles exhibit unique electronic, magnetic, catalytic and optical properties that are different from those of bulk metals. Among different metal particles, copper nanoparticles, due to their unique physical and chemical properties and low cost preparation, have been of great interest recently ${ }^{2-4}$.
The synthesis methods of these nanoparticles are basically divided into chemical, physical and biological methods. Today, it has been found, that living organisms such as bacteria, fungi, and plants have a great potential for the synthesis of metal nanoparticles ${ }^{5}$. By using these biosources various kinds of nanoparticles can be synthesized intracellularly or extracellularly which have been used for different applications. Microorganisms act as a biofactory and can also be used for the synthesis of metal nanoparticles. Majumber used a fungal species (Fusarium oxysporum) to synthesize 
copper nanoparticles (93-115 nm) at ambient temperature ${ }^{6}$. Pavani et al. used Aspergillus species of fungus for extracellular synthesis of $\mathrm{Cu}$ nanoparticles ${ }^{1}$. Lee et al. used magnolia leaf extract as reducing agent and conversion of $\mathrm{Cu}^{2+}$ to $\mathrm{CuO}$ for the synthesis of stable copper nanoparticles with a size of $40-100 \mathrm{~nm}$. They used $\mathrm{CuSO}_{4} \cdot 5 \mathrm{H}_{2} \mathrm{O}$ in aqueous solution and leaf extract to produce stable copper nanoparticles ${ }^{7}$. Valodkar et al. used stem latex of Euphorbia nivulia which is a plant with medicinal properties for the synthesis of copper nanoparticles and could synthesize Cu nanoparticles capped with peptides present in the latex of plant ${ }^{8}$. Varshney et al. used from Pseudomonas stutzeri to synthesize copper nanoparticles. They showed nanoparticles are spherical and in the range of $8-15 \mathrm{~nm}$ in dimensions ${ }^{9}$. In another work, Ramanathan et al. (2011) used a biological method to synthesize copper nanoparticles using Morganella bacteria and under aqueous physiological conditions ${ }^{10}$.

The purpose of this study was to synthesis copper nanoparticles using culture supernatant of salmonella typhimurium and characterization of the synthesized nanoparticles.

\section{MATERIALSAND METHODS}

The Bacteria culture, salmonella typhimurium was obtained from Microbiology Laboratory, Tehran University, Tehran, Iran. MullerHinton broth (MHB) was prepared, sterilized, and inoculated with a fresh growth salmonella typhimurium. The culture was centrifuged at 5000

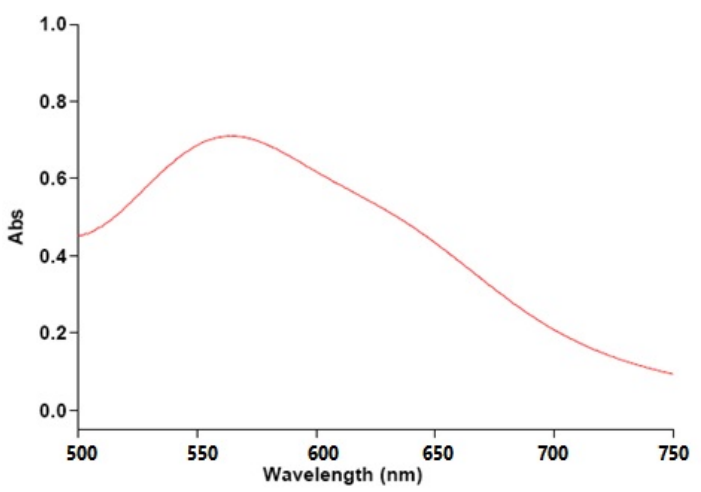

Fig. 1: UV-visible spectrum of aqueous medium containing supernatant and copper ion (1 mM) rpm for 20 minutes and the supernatant was used for the synthesis of copper nanoparticles. Distilled water was used as solvent in the synthesis of copper nanoparticles. The supernatant was added separately to the reaction vessel containing copper nitrate at a concentration of $1 \mathrm{mM}$. The reaction between this supernatant and $\mathrm{Cu} 2+$ ions were carried out for 40 minutes. The copper nanoparticles were characterized by UV-visible spectroscopy and dynamic light scattering (DLS) analysis. In addition, the copper nanoparticles were analysed by scanning electron microscopy (SEM).

\section{RESULTS AND DISCUSSIONS}

The aqueous $\mathrm{Cu} 2+$ ions were reduced during exposure to the culture supernatant of salmonella typhimurium. The color of this solution changes to cloudy orange after $40 \mathrm{~min}$ of mixing which indicated the formation of copper nanoparticles extracellularly.

Fig. 1 shows the UV-Vis absorption spectra recorded from the copper nanoparticles solution after formation. The results indicate that the reaction solution has an absorption maximum at about $565 \mathrm{~nm}$ attributed to the surface plasmon resonance band (SPR) of the copper nanoparticles. The size distribution of the synthesized nanoparticles was shown in Fig. 2. As is illustrated in figure, mean size of copper nanoparticle is about $49 \mathrm{~nm}$ and the distribution of nanoparticles is narrow $(10 \mathrm{~nm})$. Also the copper nanoparticles synthesized by salmonella typhimurium were

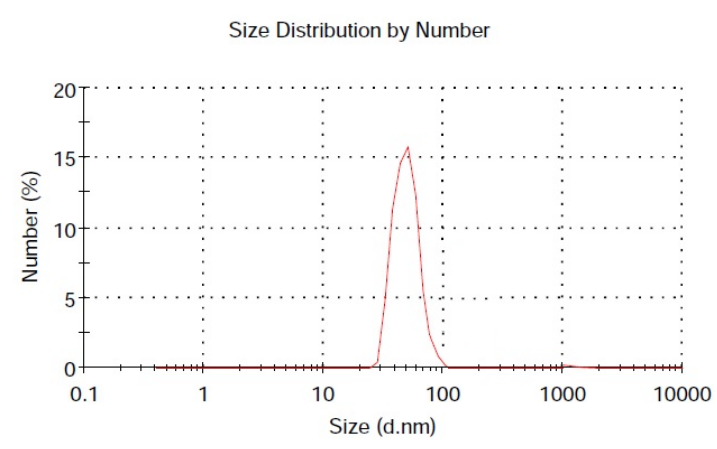

Fig. 2: The curve of size distribution (Copper nanoparticles) by number 


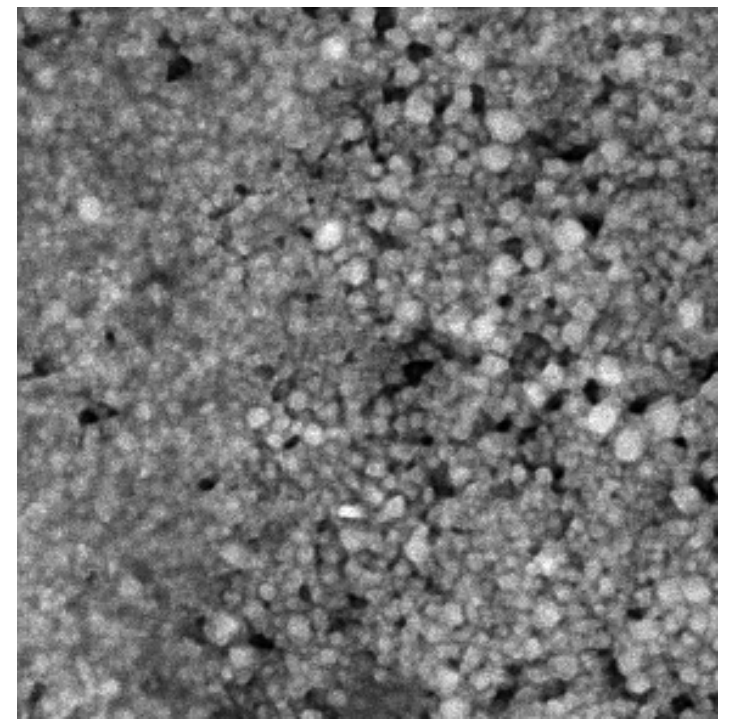

Fig. 3: SEM of copper nanoparticles produced by salmonella typhimurium studied by scanning electron microscopy (SEM) and images show and confirm copper nanoparticles production at nano-size. SEM images of the produced nanoparticles are shown in figure 3.

\section{CONCLUSIONS}

Copper nanoparticles in the range of 40 $60 \mathrm{~nm}$ are synthesized by the supernatant of salmonella typhimurium when copper nitrate is added to it. The time required to complete the copper ions reduction was obtained about $40 \mathrm{~min}$. This methodology could be used to synthesize a number of metallic nanoparticles involving other metals with good size and shape morphology. This study would therefore lead to an easy procedure for producing copper nanoparticles with the added advantage of biosafety.

\section{REFERENCES}

1. Parveen, S., Misra, R., Sahoo, SK., Nanoparticles: a boom to drug delivery, therapeutics diagnostics and imaging. Nanomed-Nanotechnol, 2012; 8(2), 147166.

2. Tavakoli, A., Sohrabi, M. \& Kargari, A., A review of methods for synthesis of nanostructured metals with emphasis on iron compounds, Chem Pap 2007; 61(3) 151170.

3. Park, B. K., Jeong, S., Kim, D., Moon, J., Lim, S. \& Kim, J. S., Synthesis and size control of monodisperse copper nanoparticles by polyol method, J Colloid Interf Sci, 2007; 311(2), 417-424.

4. Mohanpuria, P., Rana, K. N. \& Yadav, S. K., Biosynthesis of nanoparticles: technological concepts and future applications, J Nanopart Res, 2008, 10(3), 507-517.

5. Ghorbani, H.R., Safekordi, A.A., Attar, H. \& Rezayat Sorkhabadi, S. M., Biological and Non-biological Methods for Silver Nanoparticles Synthesis, Chem Biochem Eng Q, 2011, 25(3), 317-326.
6. Majumber, D.R., Bioremediation: copper Nanoparticles from electronic-waste. Inter J Eng Sci Technol, 2012, 4, 4380-4389.

7. Lee, H.J., Lee, G., Jang,N.R., Yun,J.H., Song, J.Y., Kim, B.S. , Biological synthesis of copper nanoparticles using plant extract. Nanotech. 2011; 1(1), 371-374.

8. Kuber Valodkar, M., Jadeja,R.N., Thounaojam,M.C., Devkar, R.V., Thakore,S., Biocompatible synthesis of peptide capped copper nanoparticles and their biological effect on tumor cells. Mater Chem. Phys., 2011; 128(1-2), 83-89.

9. Varshney, R., Bhadauria, S., Gaur, M.S. \& Pasricha, R., Characterization of Copper Nanoparticles Synthesized by a Novel Microbiological Method, J Met, 2010, 62(12), 100-102.

10. Ramanathan, R., Bhargava, S.K. \& Bansal, V., Biological synthesis of Copper/Copper Oxide nanoparticles, In Rose Amal (ed.) Chemeca Conference. Eng A Better World, Australia, 2011, 1-8. 